\title{
P01-1-42 Poster session
}

\section{Nanoformulated alpha-mangostin ameliorates Alzheimer's disease neuropathology by elevating LDLR expression and accelerating amyloid-beta clearance}

\author{
Xiaoling Gao, Lei Yao, Meng Hu, Xiao Gu \\ Shanghai Jiao Tong University School of Medicine, China
}

Alzheimer's disease (AD), the most common form of dementia, is now representing one of the largest global healthcare challenges. However, an effective therapy is still lacking. Accumulation of amyloid-beta (Abeta) in the brain is supposed to trigger pathogenic cascades that eventually lead to $\mathrm{AD}$. Therefore, Abeta clearance strategy is being actively pursued as a promising disease modifying therapy. Here, we found that alpha-mangostin (alpha-M), a polyphenolicxanthone derivative from mangosteen, up-regulated low density lipoprotein receptor (LDLR) expression in microglia and liver cells, and efficiently facilitated Abeta clearance. However, the in vivo application of alpha-M is limited due to its hydrophobic nature, poor aqueous solubility and stability, and thus low bioavailability and accumulation in the target organs. To overcome this limitation, alpha-M was encapsulatedinto the core ofpoly(ethylene glycol)-poly(l-lactide) (PEG-PLA) nanoparticles $[\mathrm{NP}($ alpha-M)]. Such nanoencapsulation improved the biodistribution of alpha-M in both the brain and liver, enhanced the brain clearance of 125I-radiolabeled Abeta1-42 in an LDLR-dependent manner, reduced Abeta deposition, attenuated neuroinflammatory responses, ameliorated neurologic changes and reversed behavioral deficits in $\mathrm{AD}$ model mice. These findings justified the concept that polyphenol-mediated modulation of LDLR expression might serve as a safe and efficient disease-modifying therapy for $\mathrm{AD}$ by accelerating Abeta clearance. It also demonstrated the powerful capacity of nanotechnology in modulating the biodistribution behavior of drug to improve its therapeutic efficacy in $\mathrm{AD}$. 